\title{
Hands Up: Attentional Prioritization of Space Near the Hand
}

\author{
Catherine L. Reed, Jefferson D. Grubb, and Cleophus Steele \\ University of Denver
}

\begin{abstract}
This study explored whether hand location affected spatial attention. The authors used a visual covertorienting paradigm to examine whether spatial attention mechanisms-location prioritization and shifting attention-were supported by bimodal, hand-centered representations of space. Placing 1 hand next to a target location, participants detected visual targets following highly predictive visual cues. There was no a priori reason for the hand to influence task performance unless hand presence influenced attention. Results showed that target detection near the hand was facilitated relative to detection away from the hand, regardless of cue validity. Similar facilitation was found with only proprioceptive or visual hand location information but not with arbitrary visual anchors or distant targets. Hand presence affected attentional prioritization of space, not the shifting of attention.
\end{abstract}

Keywords: attention, bimodal neurons, multisensory integration, peripersonal space, embodied cognition

In this study we investigate whether hand location directs attention to specific regions of peripersonal space (i.e., space near the body). To the human, the importance of objects in peripersonal space is that you can grasp them-and they can grasp you! They are candidates for action or potential vehicles for performing important functions. As a result, the presence of the hand near an object changes the functional implications of the object and, potentially, the need to attend to that object. If objects close to hand are represented differently from objects away from the hand, then this difference in representation could affect attention to these locations in the environment. The purpose of this article is to explore some functional consequences of bimodal, hand-centered representations in intact humans. In particular, we investigate whether spatial attention is influenced by these bimodal representations of space near the hand. Using a covert-orienting paradigm (e.g., Posner, Walker, Friedrich, \& Rafal, 1987), we examine whether hand location affects two spatial attention mechanisms: the prioritization of space, and the shifting of attention.

In the brain, peripersonal space is represented differently from space away from the body. In addition, space near the hand is represented differently from other regions of space (e.g., Graziano \& Gross, 1993, 1994, 1995; Ládavas, Pellegrino, Farné, \& Zeloni, 1998; Ládavas, Zeloni, \& Farné, 1998). Researchers have used physiological recordings from nonhuman primates to explore these differences. Rizzolatti, Gentilucci, and Matelli (1985) found that macaques with unilateral lesions to the frontal eye field failed to attend to stimuli presented in contralesional space beyond their reaching distance. In contrast, macaques with unilateral lesions to the parietal lobe failed to attend to stimuli presented within reach-

Catherine L. Reed, Jefferson D. Grubb, and Cleophus Steele, Department of Psychology, University of Denver.

We thank Elizabetta Ládavas, Alessandro Farné, Rob Roberts, and John Garza for their insights and comments regarding this work. In addition, we thank Ryan Betz and Joshua D. Baker for their help in data collection.

Correspondence concerning this article should be addressed to Catherine L. Reed, Department of Psychology, University of Denver, 2155 South Race Street, Denver, CO 80208. E-mail: creed@du.edu ing distance. These results suggest that space at different functional distances is represented by at least partially separate neural systems. Graziano and Gross (1994, 1995, 1995; Graziano, Yap, \& Gross, 1994) have proposed that combined visual and tactile representations are based on the body part that is closest to the stimulus and that these representations aid in object manipulation. In macaques, the putamen, ventral intraparietal area (VIP), area 7b, and inferior area 6 have neurons that respond to both sensory and motor activity. These areas may be best described as sensorymotor interfaces that encode the location of sensory stimuli and generate the motor responses to those stimuli. Actions controlled by these areas include movement of the head and movement of the arm directed toward and away from the body. Sensory inputs in these areas serve to select a location on the body or in peripersonal space and to recruit neurons that control movement related to that stimulus location.

Similarly, bimodal visuotactile neurons are distinguished by their characteristic response properties in peripersonal space (Fogassi et al., 1992, 1996; Gentilucci et al., 1988; Graziano \& Gross, 1993; Graziano et al., 1994; Iriki, Tanaka, \& Iwamura, 1996; Iriki, Tanaka, Obayashi, \& Iwamura, 2001; Obayashi, Tanaka, \& Iriki, 2000; Rizzolatti, Scandolara, Matelli, \& Gentilucci, 1981; see Graziano \& Gross, 1998, for a review). First, specific populations of bimodal visuotactile neurons respond to both tactile stimulation on the hand and visual stimulation near the hand. In other words, the discharge frequency of the bimodal neurons is no different when visual stimuli are presented very close to the tactile receptive fields or when tactile stimuli are presented within the tactile receptive fields.

Second, the response of these bimodal, visuotactile neurons is largely limited to visual stimuli presented in space immediately surrounding a particular body part. The neuron population we are interested in responds differentially to visual stimuli presented near the hand. Third, some bimodal neuron populations encode space on the basis of hand-centered coordinate systems. Unlike other cells that respond to visual stimuli, the visual receptive fields of these neurons are not stationary but instead move with the hand. In other words, if the hand is moved, many of these neurons will 
not respond to a visual stimulus in the same retinotopic position but will respond to a visual stimulus presented near the hand's new location. Thus, these neurons are said to represent the position of stimuli relative to the hand. In other words, they represent an object's position in hand-centered coordinates, not in retinal- or head-centered coordinates. The response patterns of such neurons suggest that the strength of a given body-part-centered representation of an object may be influenced by the likelihood that the body part will interact with the object.

Last, the response of bimodal neurons is spatially graded. As the visual stimulus is presented increasingly further from the hand, the neurons fire progressively less. These hand-centered representations appear to be specialized for objects that are on or near the hand. In sum, these bimodal visuotactile neurons are said to integrate multimodal sensory information in near, visual, peripersonal space that surrounds specific body parts, such as the hand.

Researchers have postulated that bimodal visuotactile neurons may be involved in reaching and grasping behavior as well as in basic haptic functions and fear avoidance (e.g., Cooke \& Graziano, 2004). In terms of attention, it is important to detect an event occurring near the hand so that the appropriate action-either grasping or defense movements-can be performed. Given that the tactile receptive fields are arranged somatotopically, the associated visual receptive fields form a map of the visual space immediately around the body part that is coded in body-partcentered coordinates and not in retinal or other egocentric reference systems. In this way, the visual space surrounding the body is represented as if it were a flexible medium that deforms in a topology-preserving fashion whenever the head-arm system moves. Such a map provides the location of the visual stimulus with respect to the body surface, in somatotopic or body-partcentered coordinates. Thus, researchers have suggested that the premotor area, parietal region, and putamen form an interconnected, multimodal integration system coding peripersonal space centered on body parts.

This integration of visual and tactile information has been documented in humans as well as monkeys. Cross-modal extinction studies of patients with parietal lobe damage have provided evidence that humans have bimodal neurons that represent space near body parts in body-part-centered coordinates (di Pellegrino, Ládavas, \& Farné, 1997; Farné \& Ládavas, 2000; Farné, Pavani, Meneghello, \& Ládavas, 2000; Ládavas, 2002; Ládavas, Pellegrino, et al., 1998; Ládavas, Zeloni, \& Farné, 1998). In particular, these studies focused on patients with right hemisphere brain damage who exhibited tactile extinction, that is, the inability to bring a contralesional stimulus into awareness when a competing ipsilesional stimulus is processed simultaneously. Patients could detect tactile stimuli presented on either the right or the left hand but only felt sensation on the right, ipsilesional hand if both hands were touched simultaneously. Supporting the existence of bimodal representations of peripersonal space, these patients also demonstrated cross-modal extinction in which a visual stimulus presented near the ipsilesional hand elicited the extinction of a tactile stimulus presented on the contralesional hand. An identical visual stimulus at the same location in space did not elicit cross-modal tactile extinction in the absence of a nearby hand. Further, as the distance between the visual stimulus and the hand increased, the patients were more likely to be able to feel the sensation on the other hand (Ládavas, 2002).
An important function of these bimodal visuotactile representations is that they support the integration of visual and tactile space for spatial attention in neurologically intact humans. Spence, Driver, and colleagues (Kennet, Spence, \& Driver, 2002; Spence, Nicholls, Gillespie, \& Driver, 1998; Spence, Pavani, \& Driver, 2000) used cross-modal cuing paradigms to examine the weighting and integration of explicit sensory cues. They demonstrated that a spatial cue in one modality could direct attention to a similarly located target presented in a different modality. In a typical crossmodal cuing study, spatially nonpredictive visual cues were presented near a participant's hand and were followed by a tactile target presented either to the near hand or to the other hand. Participants responded more quickly to tactile stimulation on the near hand than on the other hand, despite visual distractors (e.g. Spence et al., 1998). Likewise, tactile cues facilitated responses to visual targets presented near the stimulated hand relative to targets presented near the other hand (Spence et al., 1998). This crossmodal cuing effect occurred whether or not the stimulated hand was visible (Kennet et al., 2002) as long as the proximity of the visual stimulus with respect to the hand was maintained. These results indicate that both the cue and the target were represented according to an integrated, multimodal, spatial reference frame. Further, these effects suggest that tactile stimulation on the hands was coded via a common neural mechanism as a visual stimulus presented near the hand. Thus, with explicit cross-modal nonpredictive cues, the cross-modal cuing studies demonstrated that manipulations that affect the firing rates of bimodal visuotactile neurons also affect the effectiveness of cross-modal cues.

The cross-modal cuing studies showed that bimodal representations could influence attention through cross-modal integration. The purpose of the current study is to determine how these bimodal representations might influence different aspects of spatial attention. The flexible orientation of attention to behaviorally relevant events is a function of great importance for adaptive interaction with the environment. Selective attention involves the selection of information that is most relevant for the task at hand or the selection of the most salient aspects of processing that need to be accomplished at a particular time (Braun, Koch, \& Davis, 2001). It facilitates processing of important information in the selected region of space at the cost of reduced processing of less relevant information in the other regions of space. Covert attention improves perceptual processing by amplifying signals associated with salient regions of space (see Pashler, 1998, for a review).

The major hypothesis presented in this article is that the presence of the hand in peripersonal space changes the spatial distribution of attention. The apportionment of attention across peripersonal space may be changed by the presence of the hand because the bimodal representation of space near the hand amplifies signals from that location, thereby increasing the potential relevance of cues and targets appearing in that space. This conception of attention suggests two ways the presence of the hand and, consequently, the bimodal visual tactile representations may influence attention. First, the presence of the hand could increase the importance of the space near the hand because the hand recruits the support of bimodal representations in addition to the relevant unimodal representations. This additional support would serve to prioritize this space and potentially lead to faster responses for any targets appearing near the hand. 
Support for the idea that bimodal representations may prioritize space comes from a study by Schendel and Robertson (2004). They studied a patient who sustained damage to his right primary visual cortex and subsequently was unable to see in his left visual hemifield. When this patient's arm was placed in his "blind" left hemifield, it improved his detection of left visual field stimuli compared with when his arm was in his lap. This advantage diminished when stimuli appeared outside of grasping distance. In contrast to the cross-modal cuing and extinction studies, these results demonstrate an effect of hand proximity on visual perception in the absence of tactile stimulation. However, it is unclear whether this patient's improved performance came from the prioritization of space or from the addition of a cross-modal expectancy.

Second, in addition to prioritizing a specific region of space, the presence of the hand could influence the expectation that a relevant object will appear in that space. Bimodal representations may alter the spatial distribution of expectancy and motivational relevance (Mesulum, 1981). This expectation should have its greatest influence on the shifting of attention. Inputs from task demands (e.g., experimental design, the predictiveness of a cue), a person's attentional set, or prior experience could hold attention to a partic- ular region of space and delay moving of attentional focus to a different region of space.

\section{Current Study}

The five experiments presented in this article investigate whether the distribution of visuospatial attention can be modulated by the presence of the hand in peripersonal space and, if so, which attentional mechanisms-prioritization of space or shifting location-are most affected. The experiments used a purely visual covert attention paradigm with highly predictive cues (Posner et al., 1987). The paradigm was biased so that the observer would rely solely on visual information unless the hand and, potentially, the bimodal representations implicitly altered the distribution of attention. In these experiments, the participant's task was to detect a visual target that could appear in one of two locations (see Figure 1). On each trial, a highly predictive visual cue (70\%) indicated the probability of the target appearing at that location. Participants responded when a target appeared at either the cued location (validly cued trial) or the uncued location (invalidly cued trial). The unique manipulation of these experiments was that participants placed one hand next to one of the target locations while

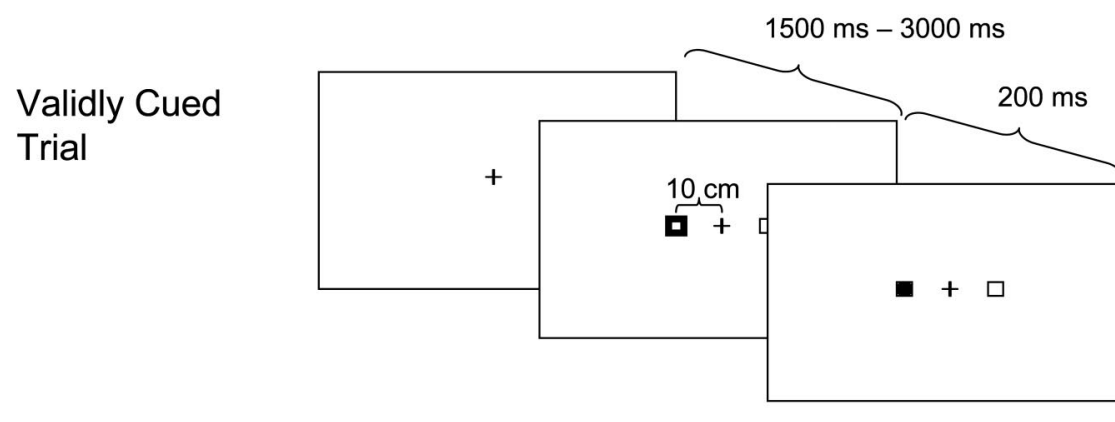

\section{Invalidly Cued \\ Trial}

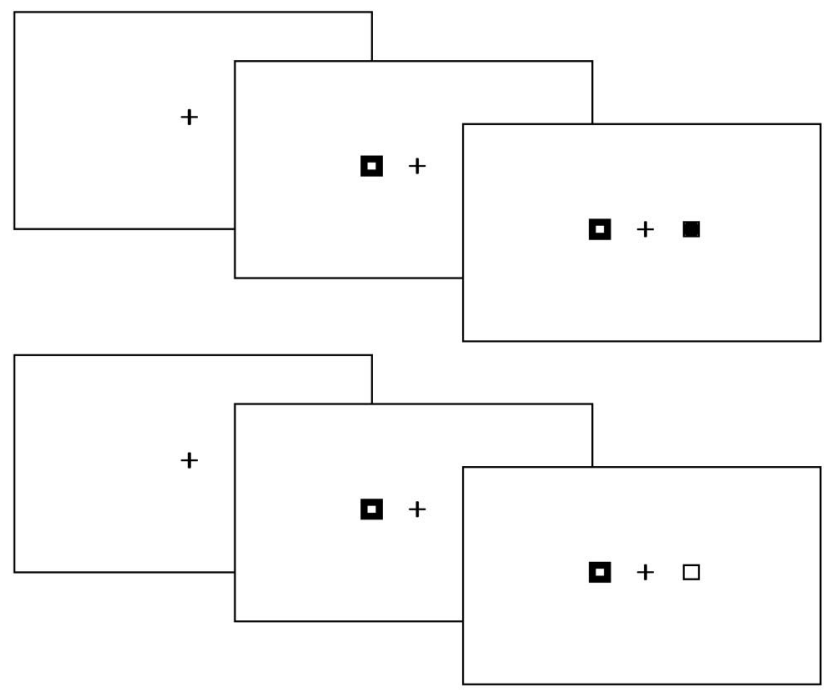

Figure 1. Covert-orienting paradigm (Posner, Walker, Friederich, \& Rafal, 1987). A fixation cross was flanked by two boxes. Attention was cued to one side by darkening the border of one box. Following the cue, a solid dot appeared in either the cued box (a validly cued trial) or in the uncued box (an invalidly cued trial). The bottom panel depicts catch trials in which a cue occurred but no target appeared in either box. 
performing this visual covert attention task (see Figure 2): They placed either their right hand next to the right-side target location or their left hand next to the left-side target location and made their detection responses with the other hand.

Unlike the cross-modal cuing studies we have described, the experiments in this study had no explicit cross-modal component, and the cues were predictive. The cues and targets were visual. Although participants had a hand next to a target location on the computer monitor, they made the target detection response on the table by the opposite hand. Thus, there was no a priori reason for the hand near the target location to influence task performance unless the presence of the hand influenced attention. As a result, these experiments are strong tests of the potential contributions from bimodal representations on attentional mechanisms. Attentional effects resulting from the combination of the location of the visual target and the location of the hand could indicate a bimodal visuotactile representation of space.

Further, this paradigm, with its predictive cues, distinguishes how hand presence and bimodal representations might affect two
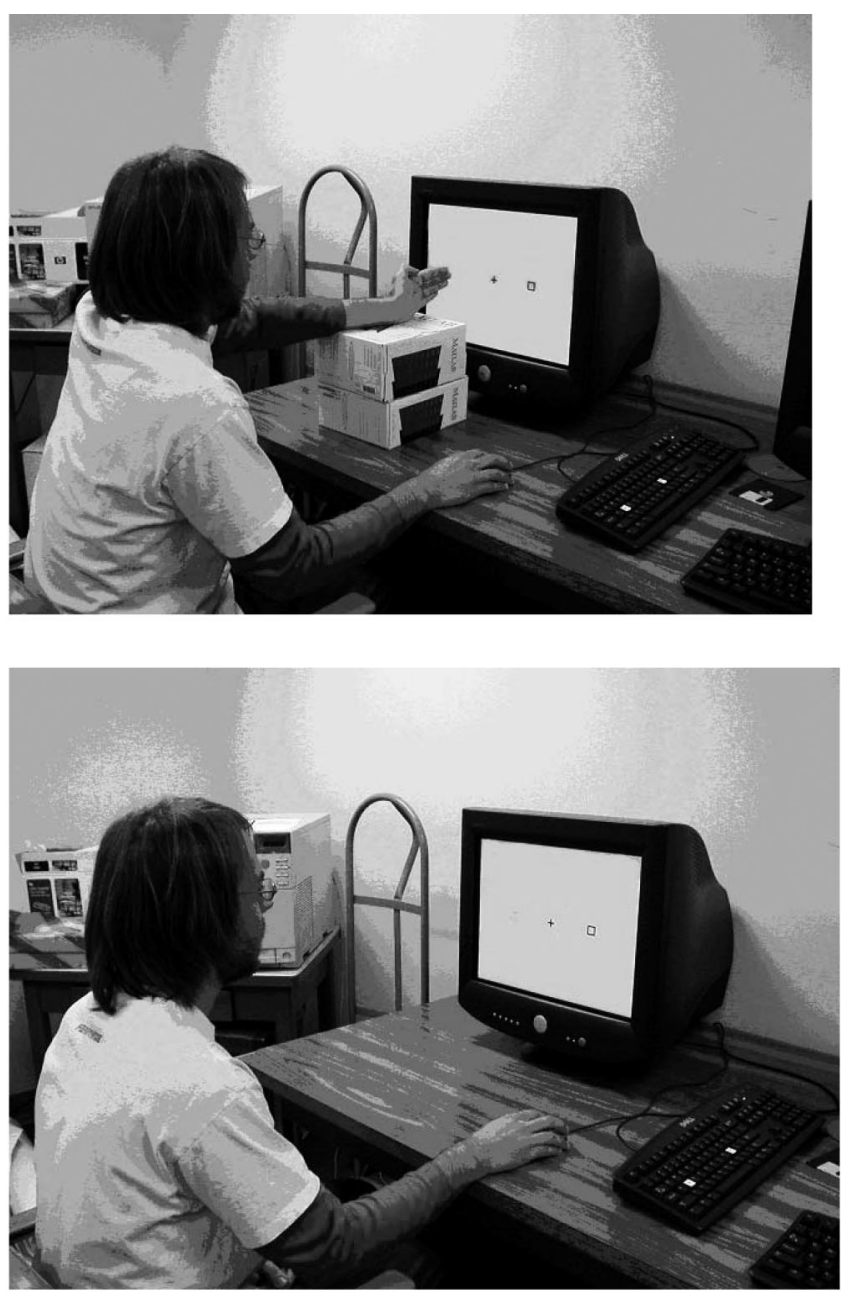

Figure 2. Experimental setup in Experiment 1. Top panel: Hand condition, in which the left hand was held next to the screen by the left box and the right hand responded. Bottom panel: No-hand condition, in which the left hand was held in the lap and the right hand responded. different aspects of attention. If hand presence influences the prioritization of space near the hand, then participants will detect targets appearing near the hand more quickly than targets appearing away from the hand. In other words, the presence of the hand will slightly bias the distribution of attention to a region near the hand. This hand facilitation effect should operate for both validly and invalidly cued targets. Alternatively, if hand presence influences the shifting of attention by changing the salience of a predictive cue, then validly cued targets appearing near the hand will have an additional advantage in processing. If this is the case, then we will observe relative response delays for invalidly cued targets appearing away from the hand. Experiment 1 explores whether hand presence influences spatial attention. Experiment 2 rules out an alternative hypothesis that any arbitrary visual anchor can change the distribution of spatial attention. Experiments 3, 4, and 5 investigate whether the influence of hand presence on spatial attention could be related to contributions from bimodal visuotactile representations. Experiments 3 and 4 explore the multimodal nature of the hand facilitation effect by selectively minimizing visual and proprioceptive-kinesthetic ${ }^{1}$ information regarding hand location. Finally, Experiment 5 determines whether the attentional effects have graded responses that grow weaker as the distance between the hand and the visual target increases.

\section{Experiment 1: Hand Presence}

Experiment 1 addresses whether the location of the body can change the distribution of attention across peripersonal space. In other words, can the bimodal representations of the space near the hand change the functional implications of that space and thereby change the relative salience of that space? To explore this question, in Experiment 1 we used highly predictive cues in a covert visual attention task (Posner et al., 1987). The task demands were unimodal in that the highly predictive visual cues provided reliable information regarding the subsequent location of the target. To determine whether the location of the hand changed the distribution of attention, we had participants put their hands next to potential target locations. We compared the hand condition with a baseline no-hand condition in which participants kept their nonresponding hand in their lap (see Figure 2). If the hand and its associated bimodal representations do not influence attention, then all the task demands are biased for visual processing, and there should be no effect on task performance with or without the hand. However, if bimodal representations influence the saliency of space near the hand despite the fact that it is irrelevant to the task, then performance should be influenced differentially by the location of the hand.

In Experiment 1 we also explore the effects of the hand on different aspects of attention-the prioritization of space and the shifting of location. In this paradigm, cues and targets appeared on left and right sides, and either the right hand was placed next to the right target location or the left hand was placed next to the left target location. If hand presence prioritized space near the hand, then we would expect a three-way interaction of hand presence,

\footnotetext{
${ }^{1}$ Throughout the article, the term proprioception refers to the combined tactile, kinesthetic, and proprioceptive inputs produced by the participant holding his or her hand against the computer screen.
} 
hand side, and target side that did not interact with cue validity. Alternatively, if hand presence affected the shifting of attention, then there should be an interaction of the validity effect with hand side and target side.

\section{Method}

Participants. Twenty-six right-handed undergraduates from the University of Denver participated for extra credit.

Stimuli and apparatus. We constructed stimuli using Microsoft PowerPoint and presented them on a $17-\mathrm{in} .(43.18-\mathrm{cm})$ monitor using E-prime 1.0 software (Psychological Software Tools, Pittsburgh, PA). Stimuli consisted of a fixation cross $\left(2^{\circ}\right)$, cue squares $\left(2^{\circ}\right)$, and a target dot $\left(1.8^{\circ}\right)$. Black stimuli appeared against a white background.

Procedure. Participants performed a standard covert attention task, as described above (see Figure 1; Posner et al., 1987). Participants were presented with a central cross, flanked by two empty squares located $5^{\circ}$ to either side. On each trial, the border of one of the two squares darkened, cuing attention to that location. On valid trials, the target (i.e., a solid black dot) appeared within the cued square. On invalid trials, the target appeared in the square on the opposite side of the fixation cross. Additionally, there were catch trials in which one square was cued but no target appeared. Within each condition of our covert orienting experiment, $70 \%$ of the trials were validly cued trials, $20 \%$ were invalidly cued trials, and $10 \%$ were catch trials.

On each trial, we asked participants to fixate on the cross. Between 1,500 $\mathrm{ms}$ and $3,000 \mathrm{~ms}$ after the beginning of the trial, one square's border darkened. On most trials, the target appeared $200 \mathrm{~ms}$ later. Participants indicated the presence of a target by pressing the mouse button on the table. We asked participants to respond as quickly and accurately as possible.

Participants completed both the left- and the right-side versions of two experimental conditions (see Figure 2): (a) no hand, in which the left hand rested in the lap and the right hand responded; (b) no hand, in which the right hand rested in the lap and the left hand responded; (c) hand left, in which the left hand was held next to the left target position and the right hand responded; and (d) hand right, in which the right hand was held next to the right target position and the left hand responded. In the hand conditions, participants held their hands thumb side up in a relaxed grasping position with their palms facing toward the center of the screen and their fingertips touching the computer monitor screen (see Figure 2). The arm was supported comfortably by a brace. There were two blocks of trials for each condition, for a total of eight blocks of 50 trials each. Block order was randomized.

\section{Results and Discussion}

For each participant, we calculated mean response times (RTs) to targets for both sides, under each cuing condition and during each hand condition. To eliminate anticipation and inattention errors, we excluded from the mean calculation trials on which participants responded incorrectly or outside a time window from $200 \mathrm{~ms}$ to $1,000 \mathrm{~ms}$ after the target appeared.

To determine whether hand presence affected attention, we conducted a repeated measures Hand Position (2: hand, no hand) $\times$ Target Side (2: left, right) $\times$ Response Hand (2: left, right $) \times$ Validity $(2 \text { : valid, invalid })^{2}$ analysis of variance (ANOVA). Confirming the basic paradigm, participants responded to valid trials more quickly than invalid trials: main effect of validity, $F(1,25)=40.73, p<.0001$. Second, participants responded to left targets in which the dominant right hand responded more quickly than to right targets: main effect of target side, $F(1$, $25)=14.73, p=.001$. Most important, a hand placed near a target position (i.e., the hand conditions) changed performance. Participants detected targets appearing near a hand relatively more quickly than targets appearing away from the hand: Hand Position $\times$ Target Side $\times$ Response Hand interaction, $F(1,25)=4.29$, $p=.049$. Post hoc analyses confirmed that this interaction between hand side and target side occurred only for the hand conditions, $F(1,24)=11.02, p=.003$, and did not occur for the baseline no-hand conditions, $F(1,24)=1.24, p=.28$ (see Figure 3 ). However, this hand-related facilitation effect occurred for both validly and invalidly cued trials: The four-way interaction that included cue validity was not significant, $F(1,25)<1$, ns. The presence of the hand did not differentially influence the shifting of attention. No other main effect or interaction approached significance $(p<.05)$.

In sum, the presence of a hand near a target affected covert orientating performance. Participants detected targets more quickly when they appeared near the hand than when they appeared away from the hand. We did not observe this same contextual bias in the baseline condition. These findings support the hypothesis that space near the hand is prioritized for attention even if the hand is not directly involved in the task in terms of either task demands (i.e., the task was purely visual) or responses (i.e., a different hand performed the response). This contextual bias is consistent with the contribution of bimodal representations to spatial attention. Although highly predictive visual cues created a strong expectancy for targets in the cued locations, the hand provided the same contextual bias for validly and invalidly cued targets. Thus, the hand influenced the prioritization of space more than the shifting of attention.

These findings concur with many current conceptions of attention in that attention facilitated the processing of some relevant objects in the environment at the cost of processing irrelevant objects (Braun et al., 2001). When the hand provided a context in peripersonal space, objects appearing near the hand were facilitated relative to objects appearing away from the hand. Hand presence made objects appearing near the hand more relevant. Nonetheless, this experiment does not rule out the possibility that any arbitrary visual anchor could provide a relative attentional advantage for objects appearing near it. We investigate this alternative explanation in Experiment 2.

\section{Experiment 2: Visual Anchor Effects}

In Experiment 2 we investigate whether the attentional facilitation of hand proximity is a result of hand presence per se (i.e., specific to the hand) or whether any similarly sized and placed

\footnotetext{
${ }^{2}$ To investigate an effect of hand proximity on spatial attention, we must demonstrate a Target Side (i.e., side where target appears) $\times$ Hand Side (i.e., side where the hand is held) interaction in which RTs are facilitated when the target appears on the same side as the hand relative to targets appearing away from the hand. However, in Experiment 1, the no-hand condition did not have a hand side. As a result, we used a response hand factor to indicate what the hand and no-hand conditions have in common (i.e., the hand that presses the mouse button). Thus, the response hand factor was a proxy for hand side in the hand condition: A right response hand refers to the condition in which the left hand was placed next to left side of the display, and a left response hand refers to the condition in which the right hand was placed next to the right side of the display.
} 


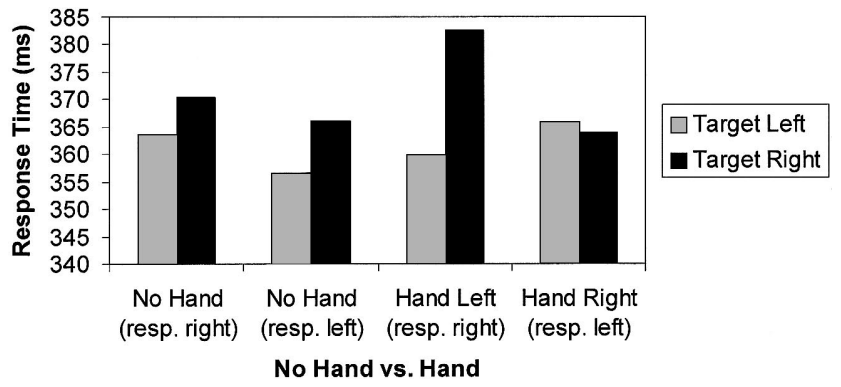

Figure 3. Experiment 1: Hand presence. Response time performance for the no-hand and hand conditions. Participants detected targets near the hand more quickly than targets appearing away from the hand. The baseline no-hand condition did not show this reversal of bias. resp. $=$ respond.

visual anchor can produce the effect. In other words, the presence of any additional visual information may draw attention to its location. We compared performance for conditions in which participants held their hand to the screen or a visual anchor was placed next to the screen. Again, an interaction between anchor side and target side, demonstrating facilitated response times for targets appearing near the anchor, would indicate that the anchors prioritized space. The interaction of anchor side and target side with validity would indicate that the anchors may also influence shifts in attention.

\section{Method}

Participants. Twenty-seven right-handed undergraduates from the University of Denver participated in this study for extra credit.

Stimuli and apparatus. We used the same stimuli and apparatus as in Experiment 1. In addition, we created a visual anchor that was approximately the same size as the hand and arm. A board that was 2 in. $(5.10 \mathrm{~cm})$ high, 4 in. $(10.16 \mathrm{~cm})$ wide, and $2 \mathrm{ft}(0.61 \mathrm{~m})$ long was supported by a brace and was positioned on its side, next to the box location, against the computer screen to create a visual input similar to that of the hand and arm.

Procedure. We used the same procedure as in Experiment 1, with the exception that we replaced the no-hand condition with a board condition. In the board condition, we placed a board near the screen in the same position that the participant's hand and forearm occupied for the hand conditions. Thus, there were left and right versions of two conditions: (a) board left, in which the left hand rested in the lap, the board was held up to the left target position, and the right hand responded; (b) board right, in which the right hand rested in the lap, the board was held up to the right target position, and the left hand responded; (c) hand left, in which the left hand was held near the left target position and the right hand responded; and (d) hand right, in which the right hand was held near the right target position and the left hand responded.

\section{Results and Discussion}

We computed mean RTs to targets in the same manner as in Experiment 1. To determine whether the hand-related facilitation effect could be found for an arbitrary visual anchor, we conducted a repeated measures Anchor (2: board, hand) $\times$ Target Side (2: left, right) $\times$ Anchor Side (2: left, right) $\times$ Validity (2: valid, invalid) ANOVA. As in Experiment 1, we found a validity effect, $F(1,26)=76.45, p<.0001$, and a left-side target detection bias, $F(1,26)=9.07, p=.006$. Relative to the hand conditions, participants responded more slowly to targets in the board conditions-anchor, $F(1,26)=3.47, p=.074$-particularly to invalidly cued targets in the board conditions: Anchor $\times$ Validity interaction, $F(1,26)=7.32, p=.012$. Although the Target Side $X$ Anchor Side interaction was significant, $F(1,26)=9.42, p=$ .005 , it was mediated by the Anchor $\times$ Target Side $\times$ Anchor Side interaction, $F(1,26)=6.25, p=.019$ (see Figure 4). Post hoc analyses revealed that only the hand conditions produced an anchor-related bias in that participants responded to targets appearing near the hand more quickly than targets appearing away from the hand, $F(1,26)=12.97, p<.0001$ (left targets: left hand $=353.78 \mathrm{~ms}$ vs. right hand $=358.22 \mathrm{~ms}$; right targets: left hand $=372.79 \mathrm{~ms}$ vs. right hand $=355.47 \mathrm{~ms})$. The board conditions showed no such anchor-side bias, $F(1,26)<1$, ns (left targets: left board $=364.53 \mathrm{~ms}$ vs. right board $=363.51 \mathrm{~ms}$; right targets: left board $=371.17 \mathrm{~ms}$ vs. right board $=372.34 \mathrm{~ms}$ ). As in Experiment 1, cue validity did not interact with hand side or target side, $F(1,26)<1$, ns. No other main effect or interaction approached significance $(p<.05)$.

In sum, the hand conditions of Experiment 2 replicated the hand conditions in Experiment 1. Participants detected targets appearing near the hand relatively more quickly than targets away from the hand. Despite the expectancy created by predictive cues, the facilitation effect did not interact with cue validity. Thus, hand presence altered the distribution of attention more than the shifting of attention. More important, the hand-related response facilitation found in Experiment 1 could not be attributed to the proximity of any arbitrary visual anchor to a target. We observed no contextual bias effect in the board conditions. Instead, these effects were specific to the proximity of the hand. Together, the results of Experiments 1 and 2 indicate that the proximity of a hand to a target facilitated detection.

Nonetheless, it is not sufficient to demonstrate an influence of hand presence on attention and claim that it reflects the operation of bimodal neurons. The effect must also be influenced by both somatosensory-proprioceptive inputs and visual inputs. In addition, it must be found only within the specific region around the hand for which bimodal neurons operate. Experiments 3-5 test the multimodal properties of the facilitation effect.

\section{Experiment 3: Proprioceptive Contributions}

By definition, bimodal neurons are multimodal. That is, the visual receptive fields of many of these neurons depend on the somatotopic coordinates of the specific body part rather than the

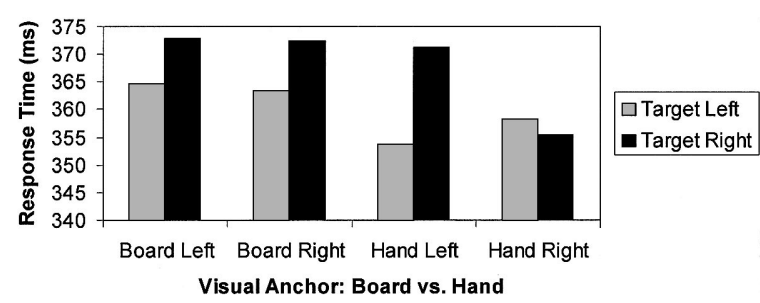

Figure 4. Experiment 2: Visual anchor effects. Response time performance for the board and hand conditions. Participants detected targets near the hand more quickly than targets appearing away from the hand. This same bias was not observed for the board (i.e., visual anchor) conditions. 
retinotopic coordinates of the target. As such, the visual response of these neurons is mediated by somatosensory-proprioceptive inputs. Thus, to test whether the attention effects of hand proximity we observed in Experiments 1 and 2 could be mediated by bimodal representations, it is necessary to demonstrate that proprioceptive inputs to hand location contributed to the facilitation effect.

Experiment 3 compares the hand conditions of Experiments 1 and 2 , in which both visual and proprioceptive information about hand location were available, with proprioceptive alone conditions, in which only proprioceptive information about hand location was available. For the proprioception alone conditions, participants held their hand in the same place and posture as in the hand conditions of Experiments 1 and 2, but their hand and arm were hidden by a box. Thus, participants could feel their hand but not see its location relative to the target. If proprioceptive information about hand location contributes to the hand-specific effect, then the hand-specific effects should occur in the absence of visual information regarding hand location. This finding would be consistent with the hypothesis that the hand-specific facilitation effect is influenced by contributions from bimodal neurons. Alternatively, if vision alone produces the effect, then it should disappear when the visual information about hand position is removed.

\section{Method}

Participants. Twenty-seven right-handed undergraduates from the University of Denver participated in this study for extra credit.

Stimuli and apparatus. We used the same stimuli and apparatus as in Experiment 1, with the exception that a box was placed over the hand and arm in the hand condition. The box was constructed out of poster board and looked like a train tunnel with a hole at one end. Participants put their arm into the hole. The box covered the hand and arm up past the elbow so that participants could feel their hand location but could not see it. Effectively, the proprioception alone condition provided identical proprioceptive information regarding hand and arm position relative to target location as the hand condition, but without the corresponding visual information.

Procedure. We used the same procedure as in Experiment 1, with the exception that we replaced the no-hand conditions with proprioception alone conditions. In the proprioception alone conditions, the participant's hand was placed near the screen, as in the hand conditions, but a box covered the hand and arm so that the participant could not see them. As a result, participants held their hand and arm in the same posture and position as in the hand condition but could only feel the positions.

Thus, there were left- and right-side versions of two conditions: (a) proprioception alone left, in which the left hand was held near the left target position behind a box and the right hand responded; (b) proprioception alone right, in which the right hand was held near the right target position behind a box and the left hand responded; (c) hand left, in which the left hand was held near the left target position and the right hand responded; and (d) hand right, in which the right hand was held near the right target position and the left hand responded.

\section{Results and Discussion}

We calculated mean RTs in the same manner as in Experiments 1 and 2. To determine whether proprioceptive inputs regarding hand location contributed to the attention effect, we conducted a repeated measures Visibility (2: proprioception alone, hand) $\times$ Target Side $(2$ : left, right $) \times$ Hand Side $(2$ : left, right $) \times$ Validity (2: valid, invalid) ANOVA. We found the validity effect, $F(1$, $26)=32.18, p<.0001$. For both the proprioception alone and the hand conditions, participants detected targets appearing near the hand more quickly than targets appearing away from the hand (see Figure 5), Target Side $\times$ Hand Side interaction, $F(1,26)=4.17$, $p=.05$. The hand and proprioception alone conditions were not significantly different for the Hand Side $\times$ Target Side interaction, $F(1,26)=4.17, p=.05$, and the attentional effect occurred regardless of validity, $F(1,26)<1$, ns. No other main effects or interactions were significant $(p<.05)$.

To determine the relative proprioceptive contribution to the facilitation effect compared with the combined proprioceptive and visual contributions, we conducted individual Target Side (2: left, right $) \times$ Hand Side $(2$ : left, right $) \times$ Validity $(2$ : valid, invalid $)$ ANOVAs. For the proprioception alone condition, the only significant effect was validity, $F(1,26)=23.23, p<.0001$. In contrast, for the hand condition, the Target Side $\times$ Hand Side interaction was significant, $F(1,26)=4.42, p=.045$, as was the validity effect, $F(1,26)=36.41, p<.0001$. Together, these results suggest that the attentional effect was more reliable for the hand condition than for the proprioception alone condition.

In sum, Experiment 3 documents the multimodal nature of the hand-specific facilitation effect. Proprioceptive input about hand location elicited the hand-related facilitation. As a result, we have discounted the hypothesis that the effect is dependent on visual hand location information. Further, the findings support the hypothesis that proprioceptive information is part of the representation. Nonetheless, the facilitation effect was more reliable when both visual and proprioceptive inputs regarding hand location were available to the participant. In sum, the presence of the hand within peripersonal space, regardless of whether the hand was actually visible, changed the prioritization of space near the hand even if it did not also affect shifts in attention.

\section{Experiment 4: Visual Sufficiency}

In Experiment 4 we investigate the contribution of visual hand position information to the attentional bias effect. Experiment 3 demonstrated that proprioceptive hand position information alone was sufficient to elicit a visual attentional bias near the hand. Thus, visual information was not necessary. The next question is whether visual information about hand position is sufficient to generate an attentional bias near the hand.

To produce visual information about hand position in the absence of proprioceptive information, we took advantage of an interesting property of bimodal neurons as well as a manipulation used by other experimenters (e.g., Farné et al., 2000; Obayashi et

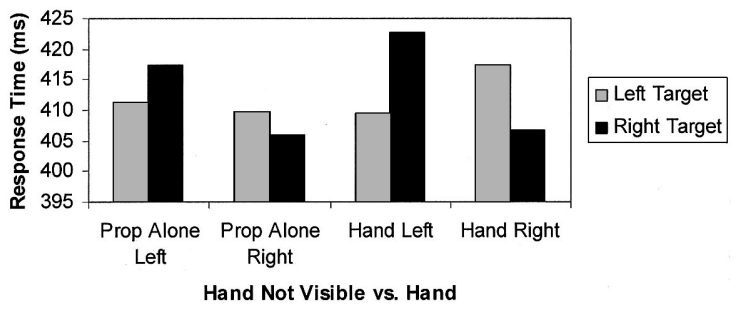

Figure 5. Experiment 3: Proprioceptive contributions. Response time performance for the proprioception alone and hand conditions. Whether or not the hand was visible, participants detected targets near the hand more quickly than targets appearing away from the hand. Prop = proprioception 
al., 2000; Pavani, Spence, \& Driver, 2000). Graziano (1999) showed that bimodal neurons in the premotor cortex of macaques responded to visual stimuli near a sufficiently convincing fake hand in the same way they responded to visual information near the macaque's real hand. Similar behavioral effects in humans have been attributed to this property of bimodal neurons. For example, Farné et al. (2000) demonstrated that brain-damaged patients with left tactile extinction exhibited cross-modal, visualtactile extinction when a visual stimulus was presented near a visible right rubber hand. In addition, Pavani et al. (2000) demonstrated the visual capture of a tactile stimulus in a target detection task if rubber hands were aligned with the position of participants' own hands.

In Experiment 4 we use a fake hand to test whether visual information about hand position in space can elicit the handspecific facilitation effect. Participants put a rubber glove on their hand, and we placed a fake rubber-gloved hand next to the box against the computer screen where the participant's own hand would have been. Thus, participants had visual information regarding hand location relative to the targets but no, or conflicting, proprioceptive information. If bimodal representations contribute to the facilitation effect, then the attentional bias should exist for the fake hand condition. This would indicate that the facilitation effect is not purely proprioceptive. However, if the facilitation effect is generated from proprioceptive input alone, then it should disappear when the proprioceptive input is removed.

\section{Method}

Participants. Twenty-six right-handed undergraduates from the University of Denver participated in this study for extra credit.

Stimuli and apparatus. We used the same stimuli and apparatus as in Experiment 1, with the exception that we used four rubber gloves- two for the left and right hands, and two for the left and right fake hands. To make more realistic-looking fake hands, we stuffed rubber gloves with cotton. We placed the fake hands next to the computer screen near the target box in the same position as the real hands. In the fake hand conditions, participants also put rubber gloves on their hands.

Procedure. We used the same procedure as in Experiment 1, with the exception that we replaced the no-hand condition with a fake hand condition. In the fake hand conditions, participants put one hand in a rubber glove and held it in their lap. The corresponding fake hand was then braced next to the screen as in the hand conditions. Thus, there were left and right versions of two conditions: (a) fake hand left, in which the left fake hand was placed near the left target position and the right hand responded; (b) fake hand right, in which the right fake hand was placed near the right target position and the left hand responded; (c) hand left, in which the left hand was held near the left target position and the right hand responded; and (d) hand right, in which the right hand was held near the right target position and the left hand responded.

\section{Results and Discussion}

We calculated mean RTs in the same way as in the previous experiments. To determine whether visual information regarding hand position was sufficient to elicit the facilitation effect, we conducted a repeated measures Fake Hand (2: fake hand, hand) $\times$ Target Side $(2$ : left, right $) \times$ Hand Side $(2$ : left, right $) \times$ Validity (2: valid, invalid) ANOVA. As before, we found a validity effect, $F(1,25)=61.85, p<.0001$. Participants responded more quickly to targets appearing near the hand or fake hand than targets appearing away from the hand or fake hand (see Figure 6), Target Side $\times$ Hand Side interaction, $F(1,25)=8.17, p=.008$. A Target Side $\times$ Hand Side $\times$ Validity interaction, $F(1,25)=6.84, p=$ .015 , indicated that the RT advantage for targets appearing near the hand over targets appearing away from the hand was stronger for invalidly cued trials. The fake hand and the real hand produced statistically equivalent attentional facilitation - that is, RTs were relatively shorter for targets appearing near both the hand and the fake hand. No other main effects or interactions were significant $(p<.05)$.

To determine the relative contributions of the fake hand to the attentional effect compared with the contributions of the real hand, we conducted individual Target Side (2: left, right) $\times$ Hand Side (2: left, right) $\times$ Validity (2: valid, invalid) ANOVAs. For the fake hand condition, the only significant effect was validity, $F(1,25)=$ $32.40, p<.0001$. In contrast, for the real hand condition, we observed significant effects for validity, $F(1,25)=73.51, p<$ .0001 ; the Target Side $\times$ Hand Side interaction, $F(1,25)=13.49$, $p=.001$; and the Target Side $\times$ Hand Side $\times$ Validity interaction, $F(1,25)=6.39, p=.018$. Together, these results suggest that the attentional effect was more reliable for the real hand than for the fake hand.

In sum, Experiment 4 confirms that visual information about hand position, without proprioceptive inputs, can elicit integrated processing of visual-tactile input regarding hand location. In particular, we have shown that when we made a connection between the participant's hand and a visual hand stimulus by using a fake hand and glove, visual input was sufficient to prioritize space near the hand. Thus, the facilitation effect was not purely proprioceptive. Further, the results support another defining characteristic of bimodal neurons, namely the integration of tactile and visual hand information.

\section{Experiment 5: Hand Proximity}

In Experiment 5 we investigate whether target distance from the hand decreases the prioritization of space near the hand and whether it modulates the shifting of attention. Bimodal neurons have a graded response in which the response rate decreases as the target moves away from the hand (Graziano \& Gross, 1995; Graziano et al., 1994; Ládavas, Pellegrino, et al., 1998; Ládavas, Zeloni, \& Farné, 1998). We used the same paradigm in Experiment 5 as in the previous experiments, but participants held their hands at the edge of the computer monitor rather than next to the target.

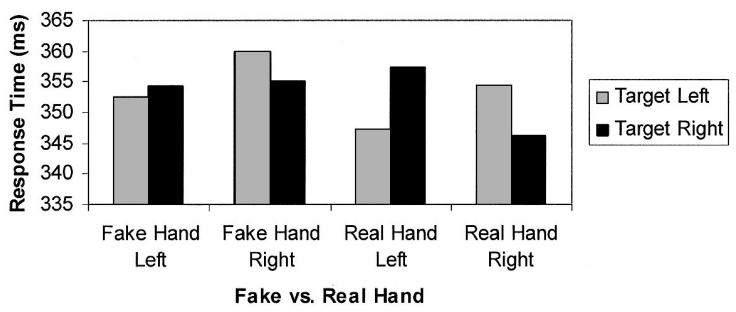

Figure 6. Experiment 4: Visual sufficiency. Response time performance for the fake hand and hand conditions. Whether or not proprioceptive information regarding hand position was available, participants detected targets near the hand more quickly than targets appearing away from the hand. 
In other words, participants held their hand at an increased eccentricity relative to the target, so that targets appeared outside of graspable space but within the same hemispace. This manipulation was different from the condition in which a validly cued target appeared on the opposite side of the screen from the hand or in contralateral hemispace. Following the predictions generated by bimodal representations, the facilitation effect near the hand should be stronger for targets appearing within the grasping space of the hand than for targets appearing outside of the grasping space of the hand. Further, a graded response would predict increased RTs for validly cued targets as the target appeared at greater distances from the hand.

\section{Method}

Participants. Twenty-six right-handed undergraduates from the University of Denver participated in this study for extra credit.

Stimuli and apparatus. We used the same stimuli and apparatus as in Experiment 1, except that we used a 20 -in. $(50.80-\mathrm{cm})$ monitor instead of a 17-in. (43.18-cm) monitor.

Procedure. We used the same procedure as in Experiment 1, with the exception that we replaced the no-hand conditions with far hand conditions. In the hand conditions, the hand was placed next to the outside of the target position, so that the target appeared close to the palm. This was identical to the hand conditions in the previous experiments. In the far hand conditions, the hand was placed $11 \mathrm{~cm}$ from the target laterally, at the edge of the monitor's screen. This distance was within a region thought to elicit responses from bimodal neurons (Schendel \& Robertson, 2004) but was outside of grasping space (i.e., where a participant could grab the stimulus). In both conditions, the hand's distance from the body was constant and the arm and hand were held in place with a brace. Thus, there were left and right versions of two conditions: (a) far hand left, in which the left hand was placed away from the target and the right hand responded; (b) far hand right, in which the right hand was placed away from the right target position and the left hand responded; (c) hand left, in which the left hand was held near the left target position and the right hand responded; and (d) hand right, in which the right hand was held near the right target position and the left hand responded.

\section{Results and Discussion}

We calculated mean RTs in the same way as in the previous experiments. To determine whether we would find a graded facilitation effect with increasing distance between the hand and target, we conducted a repeated measures Validity (2: valid, invalid) $\times$ Target Side $(2$ : left, right $) \times$ Hand Side $(2$ : left, right $) \times$ Proximity (2: hand, far hand) ANOVA. We found the expected validity effect, $F(1,25)=10.35, p=.004$. Although we found a significant Hand Side $\times$ Target Side $\times$ Validity interaction, $F(1,25)=$ $12.72, p=.001004$, it was mediated by the four-way interaction, $F(1,25)=6.35, p=.018$. In this experiment, the hand-specific facilitation was stronger for validly cued targets, especially for the near hand condition (see Figure 7). This interaction is what we would have expected if hand proximity affected attention shifts. However, this is the only experiment that produced this effect. No other main effects or interactions were significant $(p<.05)$.

To determine whether the facilitation effect for the far hand condition was weaker than for the hand condition, we conducted separate Target Side $(2$ : left, right $) \times$ Hand Side $(2$ : left, right $) \times$ Validity (2: valid, invalid) ANOVAs. For the far hand condition, only the main effect of validity was significant, $F(1,25)=9.03$,

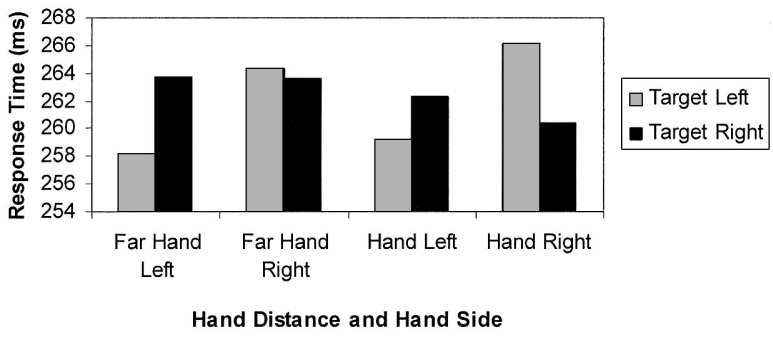

Figure 7. Experiment 5: Distance. Response time performance in the far hand and hand conditions. Although both conditions showed hand-related facilitation, the effect for the hand condition was stronger.

$p=.0006$. The Target Side $\times$ Hand Side interaction was not significant, $F(1,25)=1.22, p=.280$, nor was the three-way interaction, $F(1,25)=2.64, p=.117$. In contrast, for the hand condition, both the validity effect, $F(1,25)=9.44, p=.005$, and the three-way interaction were significant, $F(1,25)=17.58, p=$ .0001. The three-way interaction showed that the hand-related facilitation effect was stronger for validly cued targets.

To determine whether RTs for target detection decreased with distance from each hand, we conducted a repeated measures Hand (right, left) $\times$ Distance (3: hand, far hand, across screen) ANOVA on validly cued conditions. We found a significant distance effect, $F(2,24)=21.89, p<.0001$ (hand $=241.76 \mathrm{~ms}$; far hand $=$ $247.27 \mathrm{~ms}$; across screen $=262.13 \mathrm{~ms}$ ), but no main effect of hand, $F(1,25)<1$, $n s$, or interaction, $F(2,24)=2.36, p=.116$. The RTs increased with distance, but the biggest effect of distance occurred when the target appeared across the screen, completely outside of grasping space. In sum, Experiment 5 demonstrates that the hand-related facilitation effects were strongest to targets appearing within the same hemifield as the hand, which is generally consistent with the hand proximity properties of bimodal neurons.

\section{General Discussion}

In this study, we have investigated whether the location of the hand influences the distribution of attention in peripersonal space. In all five experiments, participants held their hands next to the computer screen while they performed a visual covert-orienting task; they performed the target detection response with the other hand on the table. The presence of the hand could have influenced two different aspects of attention-the prioritization of space near the hand, and the shift of attention. The results of five experiments document that participants detected targets appearing near the hand more quickly than targets appearing away from the hand, regardless of cue validity. This hand-related facilitation effect suggests that the presence of the hand prioritized the space near the hand for attentional processing. This facilitation effect was specific to the hand, given that it did not occur for an arbitrary visual anchor (i.e., a board). Further, the hand-specific facilitation effect did not interact with cue validity or the shifting of spatial attention. Thus, the context of the hand appeared to increase the relative saliency of targets appearing near the hand with a cost of processing the targets appearing away from the hand.

The specificity of the facilitation effect for space near a hand supports the hypothesis that bimodal visuotactile representations of space near the hand influence spatial attention. Neurophysio- 
logical and neuropsychological research suggest that representations of peripersonal space (i.e., space immediately next to the skin surface) may be generated from the activity of bimodal neurons that respond to both tactile and visual stimuli presented near the body (e.g., Duhamel, Colby, \& Goldberg, 1991; Graziano \& Gross, 1995; Iriki et al., 1996; Ládavas, Pellegrino, et al., 1998; Ládavas, Zeloni, \& Farné, 1998; Rizzolatti et al., 1981). Characteristic properties of these bimodal neurons include (a) responses to visual and tactile stimuli presented to the same region of the body; (b) responses limited to visual stimuli presented in the space immediately surrounding the hand, face, or body; (c) responses that decrease as distance between the visual stimulus and the specific body part increases; and (d) responses indicating that space is encoded in body-part-centered coordinate systems (i.e., the visual response is dependent on body part location rather than retinal location).

To provide evidence that bimodal representations support the hand-specific facilitation effect, in three experiments we manipulated the above properties. The hand-specific facilitation effect remained when proprioceptive but not visual information regarding hand location was available. In addition, visual information about hand location provided by a fake hand (i.e., proprioceptive information was minimized) was sufficient to produce the facilitation effect. Last, the hand-centered facilitation effect decreased when the target appeared substantially outside of the space in which the hand could grasp it. Thus, the combined pattern of results is consistent with the hypothesis that the hand-centered response facilitation effect was supported by bimodal representations.

Why might we observe attentional differences by presenting visual targets near the hand? Across experiments, peripersonal space near the hand received prioritized processing compared with other locations away from the hand. Attention is a limited resource, and objects must compete for further processing (Pashler, 1998). The context of the environment and relevance of an object for future action can increase the saliency of an expected object location. The presence of the hand might have changed the context in which the visual targets were perceived. Bimodal representations may support spatial attention by influencing the distribution of spatial attention across peripersonal space-they increase the activation or salience of the region near the hand. In addition, the location of the hand has potential functional implications for subsequent actions. Thus, objects appearing near the hand should be more salient than objects not near the hand and should be detected relatively more quickly.

This saliency for the space near the hand may arise from more than one source. One explanation of the hand-specific response facilitation is that bimodal representations provide additional neural substrates representing objects appearing near the hand compared with objects appearing far from the hand, thereby increasing the activation or saliency of that region of space. Neurophysiological evidence has demonstrated that some populations of neurons respond exclusively to visual stimuli located near the hand (Graziano \& Gross, 1995). Consequently, a visual target near the hand is represented not only by the standard visual substrates subserving visual targets but also by the additional multimodal substrates that are specific to the region around the hand. As a result, visual targets appearing in space near the hand may be represented more strongly than targets appearing far from the hand. Note that these neural substrates were different from those activated in crossmodal cuing experiments, because the present paradigm did not activate unimodal tactile neurons in either the cue or the target.

Functionally, multimodal hand-centered representations may provide additional information that is relevant to future actions. Tipper and colleagues have proposed that there are dedicated attentional mechanisms for action (e.g., Tipper, Howard, \& Houghton, 1998). Like Gibson (1979), they have argued that vision and action systems are connected functionally to promote successful interactions with the environment. Although there are many cues in the environment that engage attention and have responses associated with them, the hallmark of voluntary action is that some responses must be suppressed or inhibited to perform more relevant actions. Using several different paradigms, including interference effects (e.g., Tipper, Lortie, \& Baylis, 1992), negative priming (e.g., Tipper, 1985), and reach kinematics (e.g., Tipper, Howard, \& Jackson, 1997), Tipper and colleagues have demonstrated that when participants were given the goal to reach for an object, the frame of reference in which the objects were represented and on which selective inhibition mechanisms acted was hand centered and even action centered. For example, Tipper et al. (1992) had participants reach from a start position to press a key next to a red target light in one of three rows. At the same time, varying numbers of irrelevant yellow lights appeared. Irrelevant lights close to the hand produced more interference than irrelevant lights close to the target. The authors found the same result whether the start position was at the top row or at the bottom row. Thus, the attentional interference came from action-centered frames of reference rather than viewer-centered frames of reference.

The neural networks supporting spatial attention are well positioned to make use of the neural networks supporting peripersonal spatial representations. The ventral premotor cortex (area F4), the parietal areas (area 7b), and the putamen form an integrated (visual-tactile) network for the coding of peripersonal space centered on body parts (Colby, Duhamel, \& Goldberg, 1993; Duhamel et al., 1991; Fogassi et al., 1996; Graziano, Hu, \& Gross, 1997). It is perhaps not surprising that a similar frontoparietal network has been associated with multimodal or supramodal spatial attention in healthy humans. For example, covert orienting recruits the premotor, superior parietal, and intraparietal cortices (Corbetta \& Shulman, 1998; Macaluso, Frith, \& Driver, 2002). Further, the temporoparietal junction and inferior frontal (premotor) cortices are selectively activated for invalidly cued targets, regardless of whether the targets are visual or tactile (Macaluso et al., 2002). Similar neural regions are damaged in patients with deficits in spatial attention (Ládavas, Pellegrino, et al., 1998; Mattingley, Driver, Beschin, \& Robertson, 1997; see Pouget \& Driver, 2000).

The frontoparietal networks not only code peripersonal space and direct spatial attention (Bremmer, Schlack, Duhamel, Graf, \& Fink, 2001; Halligan, Fink, Marshall, \& Vallar, 2003) but also connect attention with action intention (Rushworth, JohansenBerg, Göbel, \& Devlin, 2003). The premotor cortex has a role in selecting movements, whereas the parietal cortex is involved with movement preparation and intention (Kalaska \& Crammond, 1995; Rushworth, Ellison, \& Walsh, 2001; Thoenissen, Zilles, \& Toni, 2002). In sum, hand-centered, multimodal representations may predispose attention to regions near the hand, potentially for future actions. 
Despite the important differences between this study and crossmodal cuing studies (e.g., Driver \& Spence, 1998; Kennet et al., 2002; Spence et al., 1998, 2000), this study extends work on multimodal contributions to visual attention. The phenomenon of visual capture was demonstrated in Experiment 4: When the apparent visual location of a body part conflicted with its actual location, vision dominated proprioception and touch. In Experiment 4, visual information regarding the location of a fake hand overrode the veridical location of participants' hands (i.e., on the lap and on the table). As a result, the visual information regarding the fake hand location, rather than the real hand location, combined with the visual information was able to elicit the facilitated target detection. Thus, the present findings are consistent with studies such as Pavani et al. (2000). Pavani et al. used a paradigm in which the visual location of a rubber hand competed with the location of a hidden, real hand. In the basic experiment, participants discriminated between upper and lower vibrotactile stimuli on their index finger and thumb, respectively, whereas visual light stimuli appeared independently in upper and lower positions. Participants' responses were relatively slowed when the visual and the tactile stimulus location were incongruent. However, when rubber hands replaced the real hands on the table holding the vibrotactile stimulators and the participants' real hands were under the table but aligned with the rubber hands, participants were likely to feel stimulation on their hands.

Nonetheless, some of the present results are different from what has been reported in the cross-modal literature. In Experiment 3, visual target detection was facilitated when only proprioceptive information about hand location was available. Although these results are consistent with the interaction of multimodal peripersonal spatial representations, spatial attention, and action systems, other studies have found that tactile and proprioceptive information regarding hand condition was not sufficient to produce multimodal effects. For example, di Pellegrino and Frassinetti (2000) reported attenuated visual extinction when a patient's fingertips were placed on the stimulus monitor adjacent to the to-be-reported visual stimulus but not when his hands were covered from view. The differences in results may be explained by a damaged brain's inability to integrate multimodal inputs. However, they may also be explained by differing visuotactile-proprioceptive task demands between the present experiments and the others. In the present experiments, vision and touch-proprioception worked together to provide convergent information about target and hand location. In the other studies, vision competed with touch or proprioception for the dominant response. As a result, the paradigm might not have cancelled out the contribution from the tactile-proprioceptive system.

In conclusion, this study considers some implications regarding how the presence of the hand in peripersonal space influences the relative distribution of spatial attention. Hand position enhanced the salience of specific regions of peripersonal space that could be relevant for future action. This spatial attention prioritization mechanism was multimodal and hand centered as well as graded in terms of proximity to the hand. Not only did humans encode nearby space through multisensory, visuotactile integrative processes, they also responded differentially to objects presented in that space. Thus, it appears that objects near the hand grab our attention.

\section{References}

Braun, J., Koch, C., \& Davis, J. L. (2001). Visual attention and cortical circuits. Cambridge, MA: MIT Press.

Bremmer, F., Schlack, A., Duhamel, J. R., Graf, W., \& Fink, G. R. (2001). Space coding in primate posterior parietal cortex. NeuroImage, 14 S46-S51.

Colby, C. L., Duhamel, J. R., \& Goldberg, M. E. (1993). Ventral intraparietal area of the macaque: Anatomic location and visual response properties. Journal of Neurophysiology, 69, 902-914.

Cooke, D. F., \& Graziano, M. S. A. (2004). Sensorimotor integration in the precentral gyrus: Polysensory neurons and defensive movements. Journal of Neurophysiology, 91, 1648-1660.

Corbetta, M., \& Shulman, G. L. (1998). Human cortical mechanisms of visual attention during orienting and search. Philosophical Transactions of the Royal Society of London, Series B, 353, 1353-1362.

di Pellegrino, G., \& Frassinetti, F. (2000). Direct evidence from parietal extinction of enhancement of visual attention near a visible hand. Current Biology, 10, 1475-1477.

di Pellegrino, G., Ládavas, E., \& Farné A. (1997, August 21). Seeing where your hands are. Nature, 388, 730.

Driver, J., \& Spence, C. (1998). Crossmodal attention. Current Opinion in Neurobiology, 8, 245-253.

Duhamel, J. R., Colby, C. L., \& Goldberg, M. E. (1991). Congruent representations of visual and somatosensory space in single neurons of monkey ventral intra-parietal cortex (area VIP) In J. Paillard (Ed.), Brain and space (pp. 223-236). Oxford, England: Oxford University Press.

Farné A, \& Ládavas, E. (2000). Dynamic size-change of hand peripersonal space following tool use. NeuroReport, 11, 1645-1649.

Farné A, Pavani, F., Meneghello, F., \& Ládavas, E. (2000). Left tactile extinction following visual stimulation of a rubber hand. Brain, 123, $2350-2360$.

Fogassi, L., Gallese, G., di Pellegrino, G., Fadiga, M., Gentilucci, G., Luppino, M., et al. (1992). Space coding by premotor cortex. Experimental Brain Research, 89, 686-690.

Fogassi, L., Gallese, V., Fadiga, L., Luppino, G., Matelli, M., \& Rizzolatti, G. (1996). Coding of peripersonal space in inferior premotor cortex (area F4). Journal of Neurophysiology, 76, 141-157.

Gentilucci, M., Fogassi, G., Luppino, G., Matelli, R., Camarda, R., \& Rizzolatti, G. (1988). Visual responses in the postarcuate cortex (area 6) of the monkey that are independent of eye position. Experimental Brain Research, 71, 475-490.

Gibson, J. J. (1979). The ecological approach to visual perception. Boston: Houghton Mifflin.

Graziano, M. A. (1999). Where is my arm? The relative role of vision and proprioception in the neuronal representation of limb position. Proceedings of the National Academy of Sciences, USA, 96, 10418-10421.

Graziano, M. S., \& Gross, C. G. (1993). A bimodal map of space: Tactile receptive fields in the macaque putamen with corresponding visual receptive fields. Experimental Brain Research, 97, 96-109.

Graziano, M. S. A., \& Gross, C. G. (1994). Mapping space with neurons. Current Directions in Psychological Science, 3, 164-167.

Graziano, M. S. A., \& Gross, C. G. (1995). The representation of extrapersonal space: A possible role for bimodal visual-tactile neurons. In M. S. Gazzaniga (Ed.), The cognitive neurosciences (pp. 1021-1034). Cambridge, MA: MIT Press.

Graziano, M. S. A., \& Gross, C. G. (1998). Spatial maps for the control of movement. Current Opinions in Neurobiology, 8, 195-201.

Graziano, M. S., Hu, X. T., \& Gross, C. G. (1997). Visuospatial properties of ventral premotor cortex. Journal of Neurophysiology, 77, 2268-2292.

Graziano, M. S., Yap, G. S., \& Gross, C. G. (1994, November 11). Coding visual space by premotor neurons. Science, 226, 1054-1057.

Halligan, P. W., Fink, G. R., Marshall, J. C., \& Vallar, G. (2003). Spatial cognition: Evidence from visual neglect. Trends in the Cognitive Sciences, 7, 125-133. 
Iriki, A., Tanaka, M., \& Iwamura, Y. (1996). Coding of modified body schema during tool use by macaque postcentral neurons. NeuroReport, 7, 2325-2330.

Iriki, A., Tanaka, M., Obayashi, S., \& Iwamura, Y. (2001). Self-images in the video monitory coded by monkey intraparietal neurons. Neuroscience Research, 40, 163-173.

Kalaska, J. F., \& Crammond, D. J. (1995). Deciding not to GO: Neuronal correlates of response selection in a GO/NOGO task in primate premotor and parietal cortex. Cerebral Cortex, 5, 1047-3211.

Kennet, S., Spence, C., \& Driver, J. (2002). Visuo-tactile links in covert exogenous spatial attention remap across changes in unseen hand postures. Perception \& Psychophysics, 64, 1083-1094.

Ládavas, E. (2002). Functional and dynamic properties of visual peripersonal space. Trends in the Cognitive Sciences, 6, 17-22.

Ládavas, E., Pellegrino, G., Farné A., \& Zeloni, G. (1998). Neuropsychological evidence of an integrated visuotactile representation of peripersonal space in humans. Journal of Cognitive Neuroscience, 10, 581-589.

Ládavas, E., Zeloni, G., \& Farné, A. (1998). Visual peripersonal space centered on the face in humans. Brain, 121, 2317-2326.

Macaluso, E., Frith, C. D., \& Driver, J. (2002). Supramodal effects of covert spatial orienting triggered by visual or tactile events. Journal of Cognitive Neuroscience, 14, 389-401.

Mattingley, J. B., Driver, J. T., Beschin, N., \& Robertson, I. H. (1997). Attentional competition between modalities: Extinction between touch and vision after right hemisphere damage. Neuropsychologia, 35, 867880

Mesulum, M. M. (1981). A cortical network for directed attention and unilateral neglect. Annals of Neurology, 10, 309-325.

Obayashi, S., Tanaka, M., \& Iriki, A. (2000). Subjective image of invisible hand coded by monkey intraparietal neurons. NeuroReport, 11, 34993505 .

Pashler, H. (1998). Attention. San Diego, CA: Psychology Press.

Pavani, F., Spence, C., \& Driver, J. (2000). Visual capture of touch: Out-of-the-body experiences with rubber gloves. Psychological Science, $11,353-359$.

Posner, M. I., Walker, J. A., Friedrich, F. J., \& Rafal, R. D. (1987). How do the parietal lobes direct covert attention? Neuropsychologia, 25, 135-146.

Pouget, A., \& Driver, J. T. (2000). Relating unilateral neglect to the neural coding of space. Current Opinion in Neurobiology, 10, 242-249.

Rizzolatti, G., Gentilucci, M., \& Matelli, M. (1985). Selective spatial attention: One center, one circuit, or many circuits? In M. I. Posner \& O. S. M. Marin (Eds.), Attention and performance XI (pp. 251-265). Hillsdale, NJ: Erlbaum.

Rizzolatti, G., Scandolara, C., Matelli, M., \& Gentilucci, M. (1981). Afferent properties of postarcuate neurons in macaque monkeys: II. Visual responses. Behavioural Brain Research, 2, 147-163.

Rushworth, M. F., Ellison, A., \& Walsh, V. (2001). Complementary localization and lateralization of orienting and motor attention. Nature Neuroscience, 4, 656-661.

Rushworth, M. F. S., Johansen-Berg, H., Göbel, S. M., \& Devlin, J. T. (2003). The left parietal and premotor cortices: Motor attention and selection. NeuroImage, 20, S89-S100.

Schendel, K., \& Robertson, L. C. (2004). Reaching out to see: Arm position can attenuate human visual loss. Journal of Cognitive Neuroscience, 16, 1-9.

Spence, C., Nicholls, M. E. R., Gillespie, N., \& Driver, J. (1998). Crossmodal links in exogenous covert spatial orienting between touch, audition, and vision. Perception \& Psychophysics, 60, 544-557.

Spence, C., Pavani, F., \& Driver, J. (2000). Crossmodal links between vision and touch in covert endogenous spatial attention. Journal of Experimental Psychology: Human Perception and Performance, 26, $1298-1319$.

Thoenissen, D., Zilles, K., \& Toni, I. (2002). Differential involvement of parietal and precentral regions in movement preparation and motor intention. Journal of Neuroscience, 22, 9024-9034.

Tipper, S. P. (1985). The negative priming effect: Inhibitory priming by ignored objects. Quarterly Journal of Experimental Psychology: Human Experimental Psychology, 37(A), 571-590.

Tipper, S. P., Howard, L. A., \& Houghton, G. (1998). Action-based mechanisms of attention. Philosophical Transactions of the Royal Society of London, Series B, 353, 1385-1393.

Tipper, S. P., Howard, L. A., \& Jackson, S. R. (1997). Selective reaching to grasp: Evidence for distractor interference effects. Visual Cognition, 4, 1-38.

Tipper, S. P., Lortie, C., \& Baylis, G. C. (1992). Selective reaching: Evidence for action-centered attention. Journal of Experimental Psychology: Human Perception and Performance, 18, 891-905.

Received September 14, 2004 Accepted June 7, 2005 